costs, because the top carbon is always the more quickly consumed.

The advantages of the arc as a source of ultraviolet light may be given as :

1. Quality and intensity of output can be varied at will by changing the electrodes.

2. No deterioration or loss of power with age.

3. A large output of warm visible light as well as ultra-violet, making the treatment pleasant.

4. Not easily broken or put out of order.

The disadvantages :

1. Uses more current than an equivalent mercury vapour lamp.

2. Carbons have to be renewed when they burn away.

3. Lamp gets hotter than mercury vapour lamp.

\section{Precautions.}

Overdosing should not be possible with lamps used for domestic self-treatment: lamps strong enough to produce overdoses should only be used under the control of a doctor.

Fire.-Lamps should be thoroughly stable and not so easily overturned that fires might be caused. All wiring and connexions should be of the best possible quality.

Electric Shocks.-Unlike the ordinary glass lamp in common use, ultra-violet lamps have one or two points where bare wires or points are exposed and may be touched when, by oversight, the current is switched on and the lamp is therefore 'alive,' although it may not be burning, and an unpleasant and even dangerous shock may thus be obtained. This may happen with the' mercury vapour lamp when cleaning the burner with alcohol, as the makers recommend, and with the carbon arc when changing or renewing the electrodes ; although, of course, both these operations should be carried out before the lamps are switched on at all. This being always a potential risk, it may be well to install lamps for home treatment in the bedroom rather than in the bathroom; this because the latter place is one in which possible shocks are far more likely to be serious on account of the large number of earthed metals and the state of moisture of floors, objects, and particularly of the body. Also, it may be well if buying an arc lamp to specify one where proper provision is made for changing electrodes without risk of shock should the switch be inadvertently left 'on.'

Care of the Eyes.-The cornea and conjunctiva are very sensitive to ultra-violet light and should always be protected by dark goggles during light treatment; these should have close-fitting side pieces.

The relative ' cost of lamps' is as follows:

Short flame Finsen are. amperes. volts.

Long flame are. .

Tungsten arc

Mercury vapour lamp .

$$
\begin{aligned}
70 \times 110 & =7700 \text { watts } \\
25 \times 110 & =2750 \quad, \\
5 \times 110 & =550 \quad, \\
4 \times 110 & =440 \quad,
\end{aligned}
$$

The carbon arcs require purchase of carbons. The tungsten arcs require expensive tungsten electrodes. The mercury vapour lamp usually wants renewing after about 600-1000 hours' run, but is the cheapest.

A self-regulating long flame arc costs about $£ 20$ $\mathfrak{E 2 5 . ~ A ~ m e r c u r y ~ v a p o u r ~ l a m p ~ w i t h o u t ~ s t a n d ~ o r ~}$ reflectors costs about $£ 10$. A hand-fed tungsten arc can be made for $\mathfrak{1 1}$ or $\mathfrak{f 2}$. Then there are the expenses of resistance coils, wiring keys, etc.

The price of lamps is put up by provision of plated reflectors and stands. A mercury vapour lamp with an iron retort stand and a tin or cardboard screen suffices. Domestic mercury vapour lamps are now sold complete for about $£ \mathrm{I0}$ direct, or $\mathfrak{E} 18$ alternating current. Hand-fed arc lamps with iron and cerium cored carbons can be had for about $£ 5$. These can be run off the house circuit.

\title{
Selection of Ultra-Violet Lamps for Home Use.
}

\section{By B. D. H. WatTers.}

$\mathrm{T}^{\mathrm{H}}$ HE idea of employing ultra-violet radiation at home is a new one, and there is as yet no wide selection of lamps made for this purpose. Those at present on the market must be regarded as largely experimental attempts to meet the new demand. The majority of them are of the type used for general irradiation in hospital practice, but reduced in size, and it is not generally realised that an exposed and live electrode which is safe enough in the laboratory or clinic in the hands of trained workers, may be quite definitely dangerous in the home.

The first of these lamps which were put on the market were all carbon or carbon-cored arcs, but recently the quartz mercury lamp has been produced commercially in a small size. The carbon lamps burn either plain carbon electrodes or carbon cored, with some metallic mixture which volatilises and adds the characteristic spectrum of that metal to that of the carbon arc. The metals or mixtures of metals selected are such as to enrich the arc with radiation of the shorter wave-lengths. From a therapeutic point of view, there seems little to choose between the different corings.

\section{Carbon Arcs.}

Ajax, Ltd.-The lamp is known as the 'Uviray,' and is a magnetically controlled tungsten-cored arc. It is remarkably steady in running owing to the automatic control. The arc is housed in a deep hood supported on a stand which contains the series resistance. A small knife switch is fitted to break the circuit, which serves the purpose admirably, but the fact that the contacts are so much exposed is a weak point in an otherwise good design. The current consumed is 4-5 amp. Price $£ 510 \mathrm{~s}$.

Apex Sun Ray, Ltd.-The No. 1 model made by this firm is a small arc made between two thin pencils of carbon which are mounted almost 
parallel in a deep metal hood. Very little adjustment is required. The series resistance is mounted on the large base of the instrument. The beam is somewhat concentrated by the narrow hood. Price $£ 66 s$.

Arnold and Son (John Bell and Croydon).-The 'Arnold' artificial alpine sun lamp is a large and expensive instrument on a heavy stand which carries two carbon arcs in series. It is made in two sizes for 5 and $10 \mathrm{amp}$. respectively. Such a lamp is more suitable for use in a hospital than in an ordinary house. Not only is the heavy current of the larger model more than the fuses and wiring of an ordinary installation can handle, but the output of radiation is more intense than is necessary for the simple tonic treatment required. Price

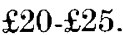

Bower Electric, Ltd.-The 'Uvral' lamp is an interesting form of double arc. The whole instrument is housed in a teak outer case, a window being raised to expose the beam. On the side of the case is a special timing switch termed an 'expometer.' This is set for some predetermined period and the lamp switched on. At the end of the period the lamp is automatically switched off, thus reducing greatly the danger of too long exposure. The general design shows that much care has been taken to avoid accidental shock. The degree of safety has to be paid for as the price is relatively high. Current 4-5 amp. Price 24 guineas.

The 'Junior' U-V ray apparatus, by the same firm, is a much smaller apparatus. Two parallel carbon pencils are mounted in the usual hood. The series resistance takes the form of spiral coils mounted round the inside of the hood. These reach a dull-red temperature when the arc is running and radiate a certain amount of heat. The lamp requires more care and attention to use than the more expensive models. Current 4.5-5 amp. Price $£ 5$ 15s. $6 d$.

Brodie, Oakley and Co._'Artsun' lamp-a handcontrolled carbon arc mounted in a reflector hood. The electrodes are narrow pencils and stick out at top and bottom of the instrument quite unpro-

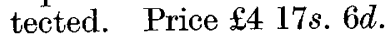

Quain Ray Lamp Co.-This is also a handcontrolled carbon or cored carbon arc, but of an unusual type. The carbons are mounted horizontally in the centre of a shallow dish-shaped reflector which is covered with a grid of stout wire. The mesh is open and does not cut down the total radiation appreciably. The series resistances are radiator heating units which are also mounted in front of the reflector, so that a certain amount of heat is radiated as well as ultra-violet radiation. A switch on the back of stand serves to shortcircuit the arc, when the lamp functions as a heating radiator of the usual type.

The carbons used are of medium size and burn fairly slowly, but the method of replacing them is clumsy and is likely to result in burnt fingers. There is also no main switch on the lamp itself for breaking the circuit. As the carbons burn away they have to be readjusted after a run of about ten minutes. This is claimed by the makers as an advantage, as it reduces the chance of an overdose owing to the patient going to sleep. At the same time, the output of radiation varies considerably during the ten minutes.

Rouse and Sons.-Rouse No. 1 Junior U.V. lamp. This is a simple carbon arc, the carbon pencils being mounted almost parallel in a metal hood supported on a light tripod. The series resistance is a separate unit. Tungsten electrodes can be obtained as well as plain carbon.

\section{Mercury Lamps.}

Medical Supply Association, Cox Cavendish Ltd., and other firms.-The 'Homesun' lamp is made with two types of burner for D.C. and A.C. In either form, the lamp is more constant than any of the carbon arcs, and the current consumption is only $2 \cdot 0-2.5 \mathrm{amp}$. The radiation is also richer in the shorter wave-lengths of the ultra-violet, but whether that is an advantage or not is a question. The D.C. burner operates better when the current is flowing in a certain direction, but, unlike the big mercury ares, it is not injured by being used with the polarity reversed. The mounting of the lamp is in a hood on a stand containing the series resistance. The heavy starting current which is characteristic of mercury lamps necessitates the use of fuses which will stand $6 \mathrm{amp}$. Price, D.C. $\mathfrak{1 1 0}$; A.C. $£ 18$.

Medical Supply Association.-The 'Medisun' lamp is a similar instrument to the above, but requiring even less current (on D.C. 1.0-1.2 amp.). An indicating 'on and off' switch is fitted. So far as running costs only are concerned, this must be the most economical source of ultra-violet radiation available at the moment. Price, D.C. $£ 10$; A.C. $£ 18$.

Stanley Cox, Ltd.-The simple type 'Actinosun' No. 2 is a mercury lamp similar to the others described, and though not made specially for use at home, would be quite suitable for that purpose. Half-power burners can be supplied. The lamp is obtainable in two models for D.C. and A.C. respectively. Price, D.C. $£ 9$ 15s.; A.C. $£ 1210 s$.

\section{Filament Lamps with Vita-Glass.}

The use of ordinary incandescent filament lamps with a vita-glass bulb for home irradiation has been somewhat superseded by these newer and more powerful lamps. The Emesay warming screen sold by the Medical Supply Association is a light radiator using ordinary bulbs, but, if the bulbs mentioned above are substituted, the screen acts as a source of weak ultra-violet of the longer wavelengths. Such an apparatus would be free from any of the risks attendant on the ignorant or incautious use of any of the lamps described above.

\section{Selecting a Home-Radiation Outfit.}

For the purchaser and the designer of ultraviolet lamps for the home, the following points should be noted:

1. The live leads, etc., should be protected by a 
deep hood or wire grid and should be as few as possible.

2. The lamp should be fitted with a good switch, in which the moving parts separate far enough to preclude all chance of arcing. A double pole type would be of advantage to ensure both electrodes being dead before they can be touched in the replacement of carbons, etc.

3. A well-fitting pair of goggles should always be worn. The use of goggles is so essential, especially with the small mercury arcs, that it would be an excellent thing if manufacturers agreed to mark all lamps intended for home use with the inscription, "Goggles must be worn when using this lamp." At present it is to be regretted that in a number of catalogues, etc., the artist has tried to express the pleasures of basking in artificial sunlight, but has omitted in his pictures the disfiguring goggles. The necessity of using goggles is mentioned in the catalogues, but a good illustration is often remembered when the written instructions have been mislaid. There are many satisfactory makes to be obtained, but, if there is any question, the only certain test is by spectrometer. A good pair of goggles should not transmit any ultra-violet radiation at all, not even of the longer wave-lengths.

The danger of falling asleep during the exposure is a perfectly definite one, as ultra-violet radiation often has that effect. In such a case it is possible for the patient to experience a severe blistering of the skin due to over-exposure, as this skin-burning does not appear until some time has elapsed. The simplest manner of guarding against this danger is to make it a rule always to stand up for an ultra. violet bath, and never to sit or lie down.

The most suitable place for the installation of these lamps is a matter for careful consideration. The bathroom, as has been pointed out elsewhere, is not the safest place, because of the danger of a bad shock due to the moisture present. On the other hand, it should be remembered that this radiation is a very powerful bleaching agent, and that the colours of fabrics, etc., will fade, and often the materials themselves perish, if exposed constantly to the rays.

The lamps reviewed here have been considered from the point of view of the man who wishes to have ultra-violet radiation available in his house as a tonic during the dark winter months. To use it for the treatment of any definite disease without the direction of a qualified physician is highly dangerous, for it must be realised that the irradiation used in medicine is often only a part of curative treatment. Further, there are conditions and diseases which can be made worse by ultraviolet radiation, and in spite of the enthusiastic pamphlets and catalogues which record its application in every human ill, it cannot be too strongly insisted that ultra-violet radiation is not a universal panacea.

\section{The Ultra-Violet Transmission of Transparent Materials.}

\section{By Dr. L. C. Martin.}

$\mathrm{W}^{\mathrm{H}}$ E may be forgiven for a little scepticism regarding the highly coloured reports which have been current during the last few years concerning the possibilities and effects of ultraviolet radiation. It must be admitted, however, that there appears to be a considerable measure of truth in such accounts; the physicist must assist in disentangling the errors and in weighing the evidence.

The shortest wave-length for which light is visible to the eye is roughly $0 \cdot 39 \mu$, but the spectrum of sunlight extends, although faintly in the end, to a wave-length of about $0 \cdot 295 \mu$ at sea-level; the cornea, the outer transparent coat of the eye, transmits down to about the sunlight limit. The 'lens' of the eye is more opaque; it will not transmit beyond $0.376 \mu$, and can be made to fluoresce by radiation of about this wave-length reaching it through the cornea. Severe inflammation of the conjunctiva can be caused by exposure to intense radiation of wave-lengths shorter than about $0 \cdot 305 \mu$. Physiological and germicidal actions occur with radiation of still shorter wave-lengths down to about $0 \cdot 21 \mu$, but beyond this point we soon reach the region $0 \cdot 193 \mu$ to $0 \cdot 185 \mu$, where air becomes practically opaque. The region of interest in ultraviolet transmission measurements for present purposes extends, then, roughly from $0.4 \mu$ to $0 \cdot 2 \mu$.

In connexion with the various sources for the production of ultra-violet radiation for clinical purposes, the transmission of the globes, screens, or containing vessels has to be studied; the transmission of window glass is also a special case. It may be added that the photometric study of the emission of the source is of no less importance. Naturally, the ultra-violet emission of many sources such as tungsten filament lamps, for which the familiar 'black-body' radiation laws are a sufficient guide, is extremely low in proportion to the total radiation. Much more energy, relatively speaking, is derived from arcs and sparks which give bright line spectra. The region $0 \cdot 4 \mu$ to $0 \cdot 2 \mu$ can be studied with the aid of fluorescence or photography. In some cases the absorption of media grows very rapidly with changing wavelength in a certain region; thus the simplest kind of information useful in some cases is the short wave-length limit of the transmission.

Quartz in the crystalline state is fairly transparent down to $0 \cdot 18 \mu$ in pieces $2 \mathrm{~cm}$. in thickness : the quartz spectrograph as made by the firms of Hilger and Bellingham and Stanley, in which the whole optical system is made in quartz, projects the entire visible and ultra-violet spectrum down to about $0 \cdot 185 \mu$ on a photographic plate; the substitution of a screen of uranium glass for the plate converts the instrument into an ultra-violet spectroscope in which the lines of the spectrum are seen by fluorescence. Wave-lengths can be given by a scale marked in the glass. An iron arc or 